\title{
Diffusion in scale-free networks with annealed disorder
}

\author{
Dietrich Stauffer ${ }^{\dagger}$ and Muhammad Sahimi \\ Department of Chemical Engineering, University of Southern California, Los Angeles, \\ California 90089-1211, USA
}

\begin{abstract}
The scale-free (SF) networks that have been studied so far contained quenched disorder generated by random dilution which does not vary with the time. In practice, if a SF network is to represent, for example, the worldwide web, then the links between its various nodes may temporarily be lost, and re-established again later on. This gives rise to SF networks with annealed disorder. Even if the disorder is quenched, it may be more realistic to generate it by a dynamical process that is happening in the network. In this paper, we study diffusion in SF networks with annealed disorder generated by various scenarios, as well as in SF networks with quenched disorder which, however, is generated by the diffusion process itself. Several quantities of the diffusion process are computed, including the mean number of distinct sites visited, the mean number of returns to the origin, and the mean number of connected nodes that are accessible to the random walkers at any given time. The results including, (1) greatly reduced growth with the time of the mean number of distinct sites visited; (2) blocking of the random walkers; (3) the existence of a phase diagram that separates the region in which diffusion is possible from one in which diffusion is impossible, and (4) a transition in the structure of the networks at which the mean number of distinct sites visited vanishes, indicate completely different behavior for the computed quantities than those in SF networks with quenched disorder generated by simple random dilution.

PACS number(s): 89.75.Da, 05.40.Fb, 82.20.-w
\end{abstract}

$\dagger$ Permanent address: Institute for Theoretical Physics, Cologne University, D-50923 Köln, Germany. 


\section{INTRODUCTION}

Scale-free (SF) networks have recently attracted much interest [1,2]. In such networks the probability distribution $f(k)$ for a node to have $k$ links to other nodes follows a power law,

$$
f(k) \sim k^{-\gamma},
$$

where $\gamma$ is a parameter that measures how well-connected the networks are. Many unusual properties of SF networks have been attributed to the distribution (1). For example, if $2 \leq$ $\gamma \leq 3$, then such networks preserve their connected structure even if their nodes are randomly removed and their structure is greatly diluted, indicating their resilience to random failure, while they exhibit vulnerability to correlated dilution whereby only the most connected nodes are removed [3-7]. In addition, Gallos and Argyrakis [8] showed that certain chemical reactions in SF networks exhibit behavior drastically different from the same reactions in regular lattices. Many properties of SF networks have been computed, including their metric [9] and percolation properties [10]. Moreover, such phenomena as epidemic spreading [11], with the intended applications being the spread of computer virus and pollution control, and the spread of extreme opinions advocated by fanatics in a society have also been studied [12] in SF networks. Most of the previous studies of SF networks employed computer simulations in order to compute various properties of interest. Hughes, Reed, and co-workers [13] developed a class of networks whose properties can be computed either exactly or by accurate mean-field approximations.

Transport processes occurring on SF networks are also of interest. For example, if SF networks are supposed to model the internet, then diffusion of particles on such networks may represent a person trying to locate information by visiting a website and its direct and indirect links, or the random spreading of a virus throughout the internet. Alternatively, such a process might also be relevant to the spread of a disease in biological networks. Diffusion (and reaction) processes have already been studied in many different systems [14-16], but studies of the properties of such processes in SF networks have started only recently [17-20]. A recent summary is given by Bollt and ben-Avraham [21].

All the studies mentioned above considered SF networks with quenched disorder. That is, once the disorder is generated in the networks, either through the power-law distribution of the connectivities, Eq. (1), or by random dilution, it remains fixed. In practice, annealed disorder one that changes with the time - may be more relevant. As an example, consider the worldwide 
web (WWW). At any given time, the links between a number of sites may temporarily be lost due to, for example, some of the sites being blocked, shut down, or hacked. The links may also be lost over a period of time because, for example, certain sites are visited so often that it becomes very difficult for a new person to visit them. Such disconnections can, at any given time, happen between a number of sites, hence giving rise to annealed disorder whereby the existence of links between various nodes may change with the time.

Even if the disorder is quenched, for application to the WWW it may be more reasonable if it is generated by a dynamical process which is happening in the network. For example, if a virus is spreading throughout the WWW, the sites that are visited by the virus and have become disabled must be removed (either temporarily or permanently) from the network. Therefore, if, for example, diffusion of particles on SF networks can represent the spread of a virus in the WWW, then studying diffusion in SF networks with annealed disorder, or quenched disorder which is generated by the random walk itself, may be more relevant to the problem.

The purpose of the present paper is to carry out a study of random walk processes in SF networks in which the disorder is either annealed, or if it is quenched it is generated by the random walk process itself. We present the results of extensive Monte Carlo simulations of diffusion in such SF networks which, to our knowledge, has never been studied before. We consider several scenarios for generating the disorder, and compute a number of important properties of diffusion in SF networks. Diffusion in regular lattices with annealed disorder was studied a long time ago [22].

The plan of this paper is as follows. In the next section we describe models of annealed disorder that we consider. Section III describes the Monte Carlo simulation procedure and the quantities that we compute. The results are presented and discussed in Section IV.

\section{MODELS OF DISORDER}

Before introducing any disorder into a SF network, we grow the network with $N$ sites such that every new site selects four of the already existing sites as neighbors, with a probability proportional to the number of neighbors the selected site had before. We start with four sites connected to each other. Such a method for generating a SF network has been used in numerous papers [1]. In such a network, the probability for a site to have $k \geq 4$ neighbours decays as $1 / k^{3}$ (i.e., $\gamma=3$ ). We do not attempt to vary $\gamma$ as a parameter of the model. We now consider 
several scenarios under which the topology of the grown network may change with the time. These are as follows.

\section{Model A}

In the first model we let the SF network change under the influence of the walkers, say a virus that is penetrating the WWW. At each time step, every site which is not yet visited is deleted permanently from the network with probability $p$. The idea is that if, for example, a virus is spreading throughout the WWW, at every time step, a certain fraction of the uninfected sites becomes aware of the existence of the virus, and take themselves out of the WWW for a certain time. Alternatively, we also study the case in which the already visited sites (representing the infected sites) are deleted in order to compare the results with the first scenario.

\section{Model B}

In this model only after visiting a site (of the previously constructed network) is attempted $m$ times, the site becomes available permanently to the walkers. The idea is that, as it often happens in practice, a site cannot be accessed by a visitor because, for example, it has been shut down for a certain period of time, or it is already being visited so heavily that it becomes much more difficult for additional visitors to access the site, or that one has forgotten the precise address of a site and, therefore, many attempts are made in order to find the correct address of the site to access it.

\section{Model C}

In this model we combine the two effects of forgetting a site and having to try repeatedly, in order to generate annealed disorder.

\section{Model D}

In this model a random walker still needs, as in model $\mathrm{C}, m$ attempts to visit a new site, but if a site is visited, then a repeat visit is possible only if the total number of attempts is at least $m+P \times \min (m, \Delta t)$, where $\Delta t$ (measured in units of the number of steps taken) is the time elapsed after the last successful visit, and $P \ll 1$ a new "forgetfulness" parameter. 
Thus, a site which is visited, but then not visited again for some time (which can be long), is treated as if it was never visited before which, from a practical view point regarding the WWW, seems reasonable. Hence, this type of annealed disorder involves a feedback mechanism between consecutive visits to the sites of a SF network, or the WWW.

\section{Model E}

This model is motivated by the fact that, if the best connected sites of a SF network are removed, most of the network's connectivity is lost [3-7]. Thus, we first remove a certain fraction of the better connected sites of the network, and then use Model D in order to generate annealed disorder and carry out simulation of the diffusion process. Therefore, Model E contains both quenched and annealed disorder.

\section{MONTE CARLO SIMULATION}

A property of random walk processes, which from a practical point of view is most relevant to SF networks and their possible applications to WWW, is the mean number of distinct sites visited after time $t, S(t)$, which characterizes the coverage of the space by the random walkers. The SF networks that we utilize are not embedded in Euclidean space. In addition, it has been shown (see Cohen and Havlin [9]) that the diameter of SF networks that we simulate (with $\gamma=3$ ), which represents the minimal number of links needed to connect two network sites, is only of the order of $\ln N$, so that, for example, the WWW with about $8 \times 10^{8}$ nodes has a very small diameter [23]. Therefore, the mean-square displacement of the random walkers is not a very useful property, because it does not measure any true distance that the random walkers travel in the space.

For some of the models we also compute the number $R(t)$ of returns to the origin of the random walks. The traditional probability $P_{0}(t)$ that the random walkers return to the origin of their walks is simply $\propto d R(t) / d t$. Also computed is the number $A(t)$ of the sites that are accessible to the random walkers at time $t$. The latter quantity is particularly important to the application involving WWW and the spread of a virus in computer networks. Most of our results were obtained using $10^{2}$ or $10^{3}$ realizations of the SF networks with up to $N=10^{6}$ sites each.

\section{RESULTS AND DISCUSSIONS}


In the first series of simulations, we put one random walker onto one of the four initial sites of the SF network, and at each time step $t$ the walker selects with probability $1 / 2$ one of its $k$ neighbours and moves there. Each connection between two neighboring sites can be travelled in both directions. If, instead, we take directed or hierarchical SF networks with one-way links (so that a hop from one site to another is allowed only in a fixed direction), the walkers would soon be trapped. We average for each realization the results over the four starting points, and then over all network realizations.

Figure 1 (top) indicates that the mean number of distinct sites visited is given by

$$
S(t) \propto t
$$

for intermediate times (see also Gallos [18]). Equation (2) is not valid for short times. For long times finite-size effects dominate and one obtains $S=N$, i.e., every site of the network will eventually be visited. The fraction $S(t) / N$ of the visited sites is a size-independent function of $t / N$ for not too short times. This is shown in Fig. 1 (bottom) which indicates that

$$
S(t) / N=f(t / N)
$$

Scaling law (3) is analogous to opinion dynamics [24], where the number $S(t)$ of surviving opinions equals the number $N$ of possible opinions, multiplied by a scaling function $f(t / N)$, where $t$ represents the number of people. The linear dependence of $S(t)$ on $t$ is similar to what one obtains for random walks on the Bethe lattices or Cayley trees, branching structures in which every node is connected to a fixed number $k$ of other nodes with no closed loops allowed. It was shown by Hughes and Sahimi that for such trees [25]

$$
S(t) \sim \frac{k-2}{k-1} t
$$

Hughes, Sahimi, and Davis [25] showed that even if small closed loops are allowed to form in the tree structure, the essence of Eq. (4), namely, the linear dependence of $S(t)$ on $t$, would remain unaltered.

Figure 2 shows the results for $S(t)$, computed for Model A, for several values of the blocking probability $p$. For better efficiency in the simulations we delete only sites which are just visited by the ant, with probability $p t$ instead of $1-(1-p)^{t}$. Thus, at time $t=1 / p$ the annealed disorder no longer has any effect and the network is quenched. The results indicate that the 
annealed disorder generated by blocking has an effect similar to a priori restriction of the network size shown in Fig. 1. It does not matter much whether all sites, or only previously unvisited sites, can be removed.

Figure 3 presents the results for $S(t)$, computed for Model B, using several values of the parameters $m$, the number of attempts to visit a site before that site becomes available. The results indicate the strong delay in the number of distinct sites visited, especially for large values of $m$. In particular, for short and intermediate times $S(t)$ varies with $t$ nonlinearly and in a nontrivial manner. Eventually, however, the linear growth of $S(t)$ with $t$ seems to set in.

The results for $S(t)$ for Model C, in which we combine the disorder in Models A and B, are shown in Fig. 4 (top). Even for an extremely small and fixed value, $p=10^{-5}$, diffusion becomes increasingly difficult as $m$ increases. Strong delays in the growth of $S(t)$ are seen even for $m$ as small as 5. For $m=50$, the largest value that we considered, there is a long delay in the growth of $S(t)$ with $t$, followed by a nonlinear growth. At long times, $S(t)$ more or less saturates at values much smaller than $N$, the total number of sites in the network. Similarly, for a fixed $m$ as small as 2 , diffusion becomes impossible for $p$ as small 0.1 . Even for $p=10^{-2}$ the walker visits very few sites before its motion becomes incapable of taking it to new sites. These results are also shown in Fig. 4 (bottom).

Figure 5 presents a phase diagram for Model D, indicating that for a certain region in the $(m, P)$ space, diffusion becomes impossible and the walkers become blocked. The curve that separates the diffusion/no diffusion regions is given roughly by, $m=1 / P$, so that for $m>1 / P$ no diffusion is possible and the random walkers remain at their starting place. For $m<1 / P$ the results agree with those for $P=0$, when the model becomes equivalent to Model B.

Figure 6 , showing the average number $R(t)$ of visits to the origin, indicates that in Model D the random walkers return to their starting points very rarely. Even at relatively long times, very few visits to the origin take place and, in fact, with increasing $m$ the visits become more rare, since more sites are unavailable at any given time. The traditional probability $P_{0}(t)$ of return to the origin that has been calculated for many random walks is simply the derivative with respect to $t$ of the curves shown in Fig. 6, which would indicate a sharp decay of $P_{0}(t)$ with $t$ (not shown).

An important quantity is the number of nodes that are accessible to a random walker during its motion. For example, if a virus is moving through the WWW, the number of nodes that it 
can potentially reach in order to infect them determines the "success" of the virus. We have computed this quantity for Model D; the results are shown in Fig. 7 (top), where we show the dependence of the number of accessible sites on the time $t$ for several network sizes, with $P=0.1$ and $m=5$. Qualitatively, the results are similar to the number of distinct sites visited, although numerically they are quite different. For intermediate times, the number of accessible sites follows a scaling law similar to Fig. 1 for the number of visited sites. This is demonstrated in Figure 7 (bottom).

Finally, for Model E, we remove permanently a fraction $q$ of the $N$ network sites and study, using Model D, how many sites can still be visited for $t \rightarrow \infty$. If the deletion is random, $q$ must be close to unity to split the network into small parts [3]; if the $q N$ most connected sites are removed, then a small $q$ is sufficient to split the network [5]. We consider none of such extreme limits, but instead remove the first $q N$ sites which joined the network during its creation. They are usually the most connected ones, but exceptions do exist. Moreover, the initial core of four site belongs to the $q N$ deleted sites. Thus, the random walkers start their diffusion on such sites but can never return there. Figure 8 suggests a phase transition at intermediate values of $q$, from a large connected remaining network at small $q$, to a fragmented assembly of much smaller clusters at large $q$, as in percolation on square lattices. Similar results, but with much less precise statistics, were obtained for the number of accessible sites (not shown).

Let us emphasize that none of the results presented in this paper are obtained when one considers random walks in SF networks with quenched disorder generated by simple random dilution (as opposed to being generated by the dynamical process itself). We believe that the results presented in the present paper indicate not only the significance of the disorder of the type we consider, but also their potential applicability to networks encountered in practice, and in particular the WWW which contains either annealed disorder, or quenched disorder which is, however, generated by a dynamical process.

We thank L. Gallos for helpful information.

\section{SUMMARY}

We carried out extensive Monte Carlo simulation of diffusion in scale-free networks with either annealed disorder, or quenched disorder which, however, is generated by the diffusion process itself. Several models of disorder, motivated by application to the worldwide web, were 
considered and important properties of the diffusion process, such as the number of distinct sites visited and the fraction of accessible sites to the walkers at any given time, were computed. The results indicate their drastic departure from those in SF networks with quenched disorder generated by simple random dilution. For some of the models we considered, scaling laws for the effects of finite times and finite sizes were demonstrated. For the more complex models of disorder, where the structure of the available network is determined by the diffusion process itself, diffusion may become impossible in some parameter range, hence providing potential strategies to handle certain phenomena in SF networks and the WWW, such the spread of a virus in such networks. 
[1] R. Albert and A.L. Barabási, Rev. Mod. Phys. 74, 47 (2002).

[2] J.F.F. Mendes and S.N. Dorogovtsev, Evolution of Networks: From Biological Nets to the Internet and the $W W W$ (Oxford University Press, London, 2003).

[3] R. Cohen, K. Erez, D. ben-Avraham, and S. Havlin, Phys. Rev. Lett. 85, 4626 (2000).

[4] R. Albert, H. Jeong, and A.-L. Barabási, Nature (London) 406, 378 (2000).

[5] R. Cohen, K. Erez, D. ben-Avraham, and S. Havlin, Phys. Rev. Lett. 86, 3682 (2001).

[6] Y. Moreno, J.B. Gómez, and A.F. Pacheco, Europhys. Lett. 58, 630 (2002).

[7] L. Gallos, R. Cohen, P. Argyrakis, A. Bunde, and S. Havlin, Phys. Rev. Lett. 94, $188701(2005)$.

[8] L. Gallos and P. Argyrakis, Phys. Rev. Lett. 92, 138301 (2004).

[9] A.F. Rozenfeld, R. Cohen, D. ben-Avraham, and S. Havlin, Phys. Rev. Lett. 89, 218701 (2002); C.P. Warren, L.M. Sander, and I.M. Sokolov, Phys. Rev. E 66, 056105 (2002); R. Cohen and S. Havlin, Phys. Rev. Lett. 90, 058701 (2003); G. Bianconi and A. Capocci, Phys. Rev. Lett. 90, 078701 (2003).

[10] S.N. Dorogovtsev, J.F.F. Mendes, and A.N. Samukhin, Phys. Rev. E 64, 066110 (2001); N. Schwartz, R. Cohen, D. ben-Avraham, A.-L. Barabási, and S. Havlin, ibid. 66, 015104 (2002); R. Cohen, D. ben-Avraham, and S. Havlin, ibid. 66, $036113(2002)$.

[11] R. Pastor-Satorras and A. Vespignani, Phys. Rev. Lett. 86, 3200 (2001); D. Volchenkov, L. Volchenkova, and Ph. Blanchard, Phys. Rev. E 66, 046137 (2002); L.K. Gallos and P. Argyrakis, Physica A 330, 117 (2003).

[12] D. Stauffer and M. Sahimi (unpublished), physics/0506106 and 0506154. 
[13] W.J. Reed and B.D. Hughes, J. Theor. Biol. 217, 125 (2002); Phys. Rev. E 66, 067103 (2002); Physica A 319, 579 (2003); D.Y.C. Chan, B.D. Hughes, A.S. Leong, and W.J. Reed, Phys. Rev. E 68, 066124 (2003).

[14] B.D. Hughes, Random Walks and Random Environments, Volume 1 (Oxford University Press, London, 1995).

[15] D. ben-Avraham and S. Havlin, Diffusion and Reactions in Fractals and Disordered Systems (Combridge University Press, Cambridge, 2000).

[16] M. Sahimi, Heterogeneous Materials I (Springer, New York, 2003), Chapters 5 and 6.

[17] J.D. Noh and H. Rieger, Phys. Rev. Lett. 92, 118701 (2004).

[18] L.K. Gallos, Phys. Rev. E 70, 046116 (2004).

[19] T. Di Matteo, T. Aste, and M. Gallegati, physics/0406091.

[20] I. Simonsen, K.A. Eriksen, S. Maslov, and K. Sneppen, Physica A 336, 163 (2004).

[21] E.M. Bollt and D. ben-Avraham, New J. Phys. 7, paper 26 (2005).

[22] M. Sahimi, J. Phys. C 19, 1311 (1986).

[23] R. Albert, H. Jeong, and A.-L. Barabási, Nature (London) 401, 130 (1999).

[24] D. Stauffer, A.O. Sousa, and C. Schulze, J. Artificial Societies and Social Simulation (jasss.soc.surrey.ac.uk) 7 (No. 3), paper 7; S. Fortunato, Int. J. Mod. Phys. C 15, 1021 (2004); F. A. Rodrigues and L. da F. Costa, Int. J. Mod. Phys. C 16 (No. 11) (2005).

[25] B.D. Hughes and M. Sahimi, J. Stat. Phys. 29, 781 (1982); B.D. Hughes, M. Sahimi, and H.T. Davis, Physica A 120, 515 (1983). 

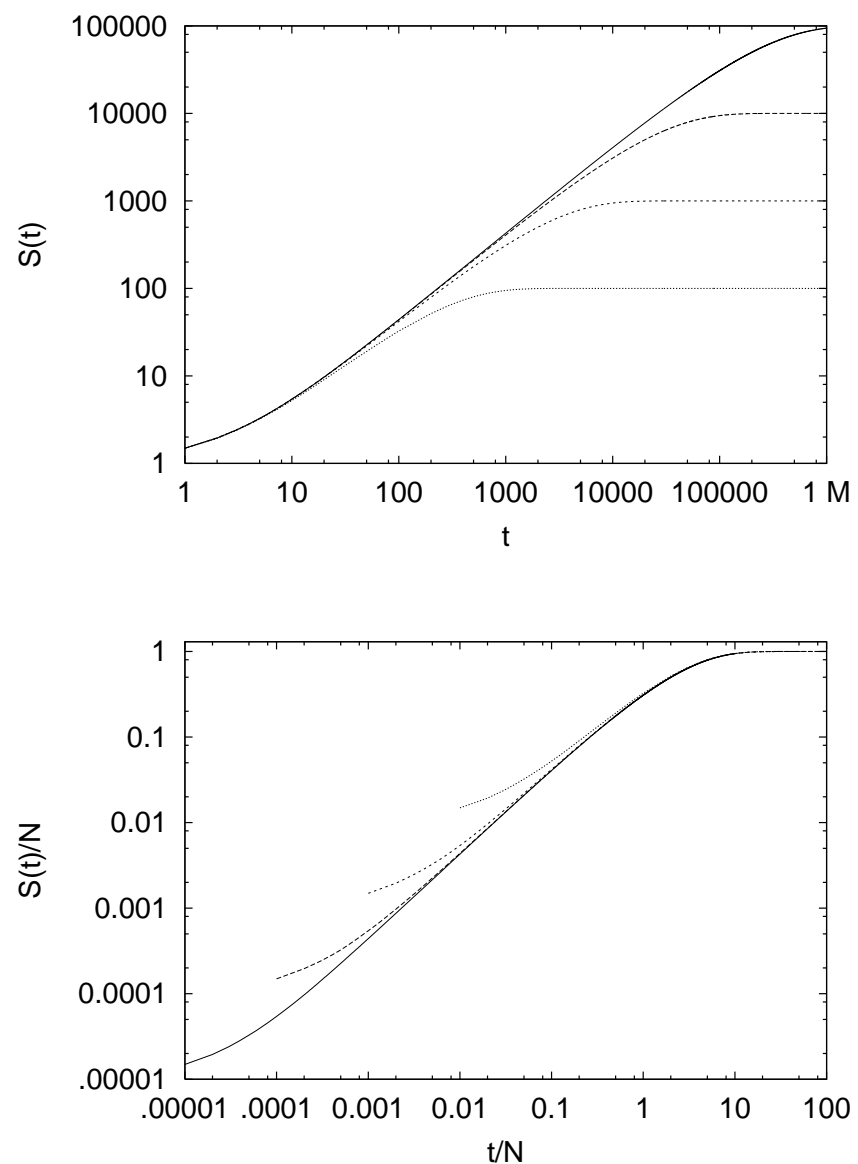

Figure 1: Top: Time-dependence of the number of distinct sites visited, averaged over $10^{3}$ samples of four walkers each. The static network has, from top to bottom, $N=10^{5}, 10^{4}, 10^{3}$, and $10^{2}$ sites. Bottom: Scaling representation of the results in the top figure, with $N$ increasing from right to left. 

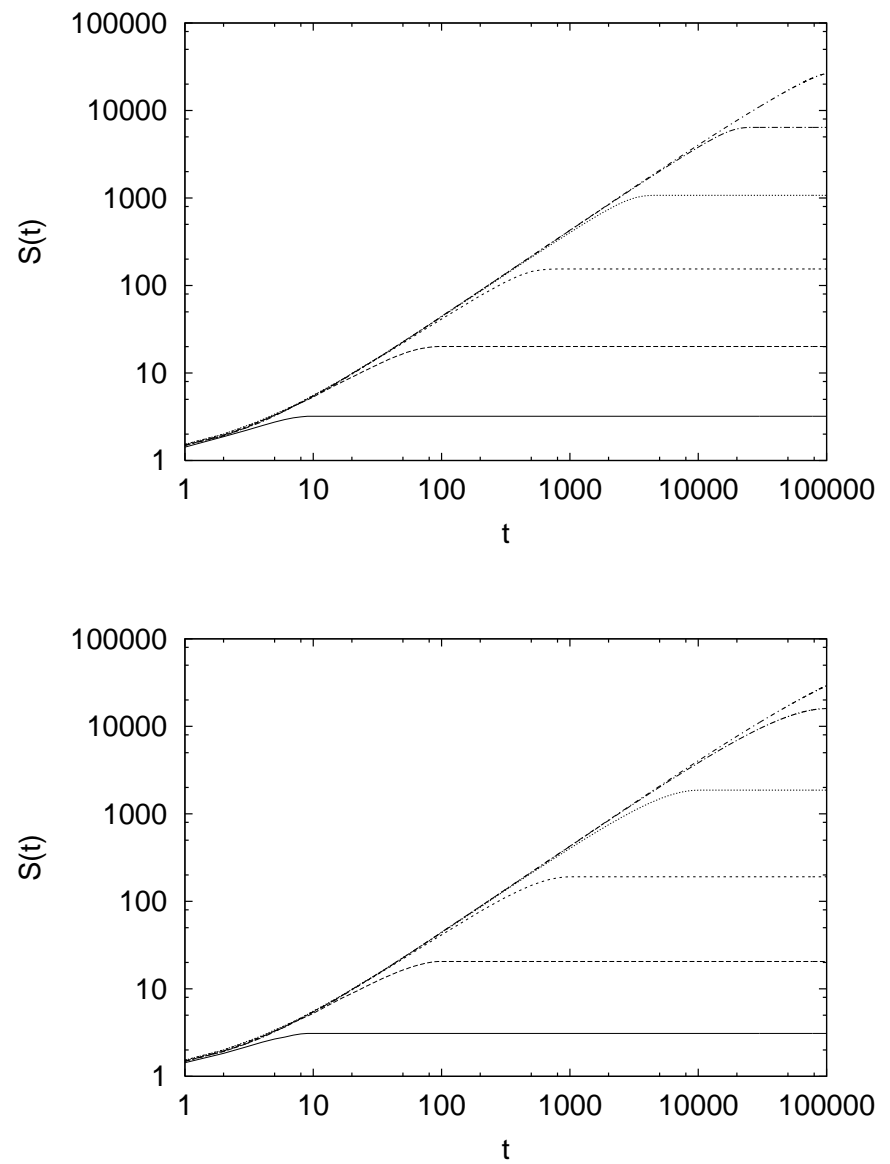

Figure 2: Number of distinct sites visited in Model A as a function of time $t$, averaged over 100 samples of four walkers each. The network contains disorder with a fraction $p t$ of the sites not available at time $t$ with, from top to bottom, $p=10^{-6}, 10^{-5}, 10^{-4}, 10^{-3}, 10^{-2}$, and $10^{-1}$. In the top part, all sites can be deleted, in the bottom part only the unvisited sites. 


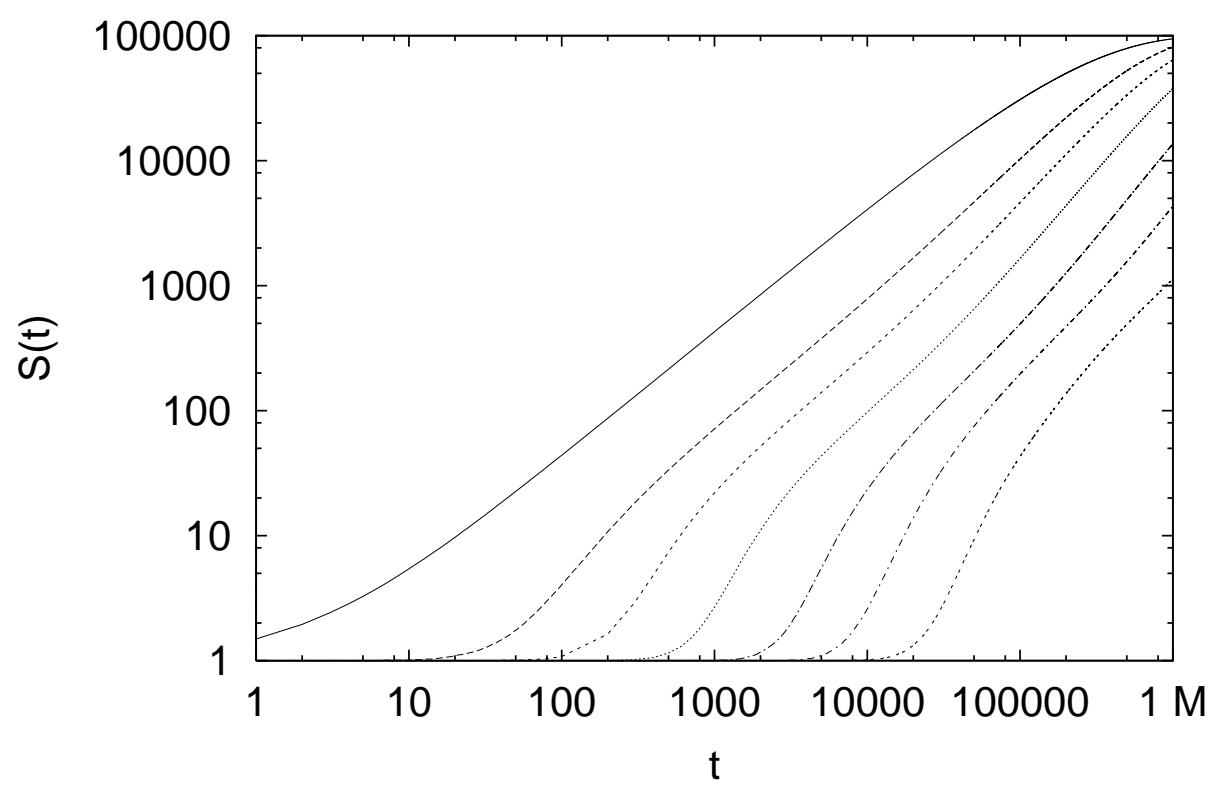

Figure 3: Number of distinct sites visited in Model B. The results are for, from left to right, $m=1$ (same as in Fig. 1), 2, 3, 5, 10, 20, and 50, all computed for networks of size $N=10^{5}$ and averaged over $10^{3}$ samples. 

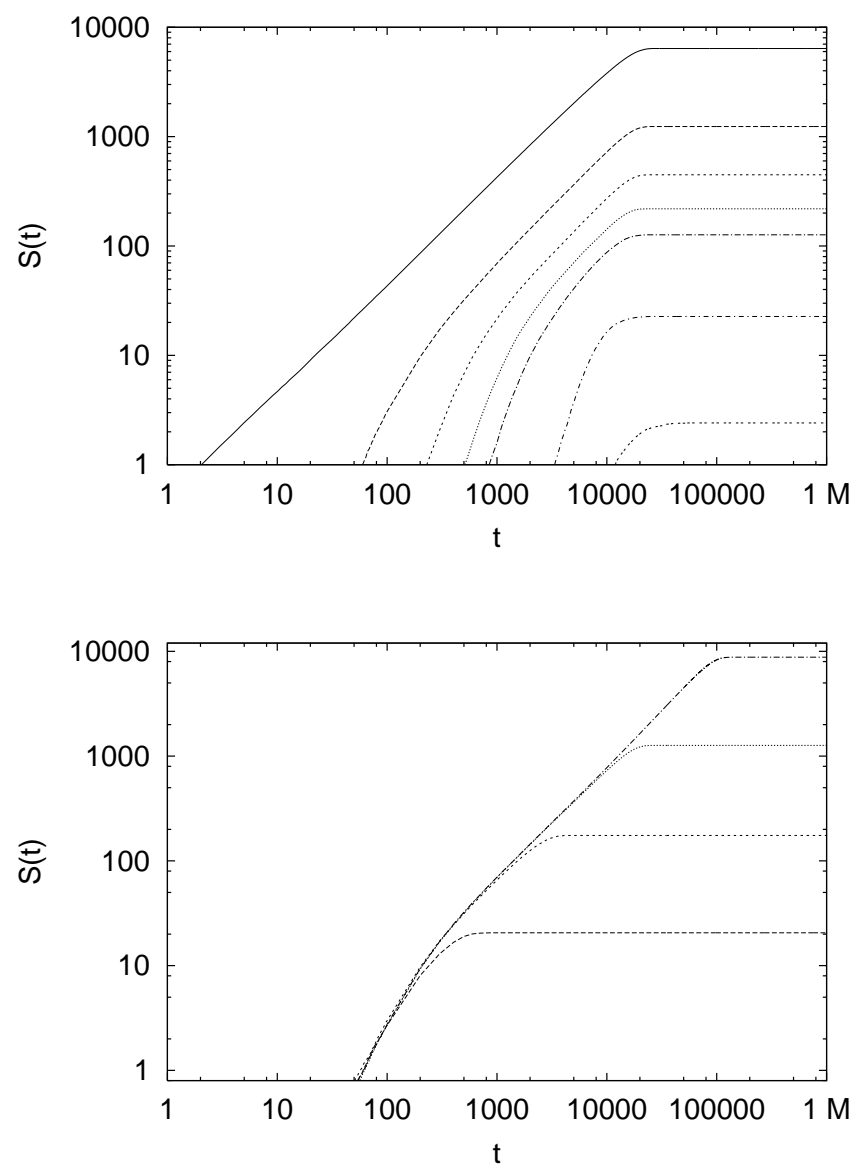

Figure 4: Top: Number of distinct sites visited, averaged over 100 samples, in Model C with $p=10^{-5}$ and, from left to right, $m=1,2,3,4,5,10$, and 20 . (The results for $m=50$ are too small to be seen; here we do not count the starting point as being visited at time zero.) Bottom: Results for, from bottom to top, $p=10^{-3}, 10^{-4}, 10^{-5}$, and $10^{-6}$ and $m=2$. For $p \geq 0.1$ no diffusion was possible, while $S(t)<1$ for $p=0.01$. 


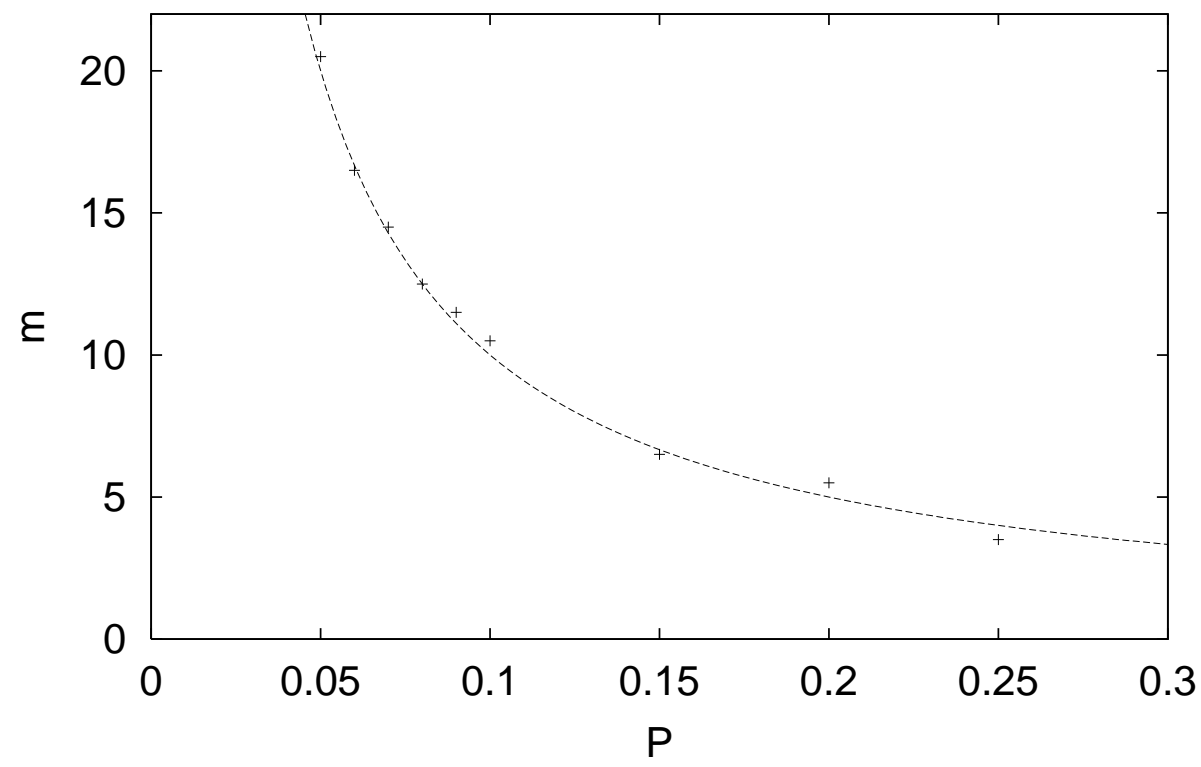

Figure 5: Phase diagram for Model D. For pairs $(m, P)$ that are above the curve diffusion is impossible. The results are averages over 10 or 100 realizations of $N=10^{4}$. The curve shown is $m=1 / P$.

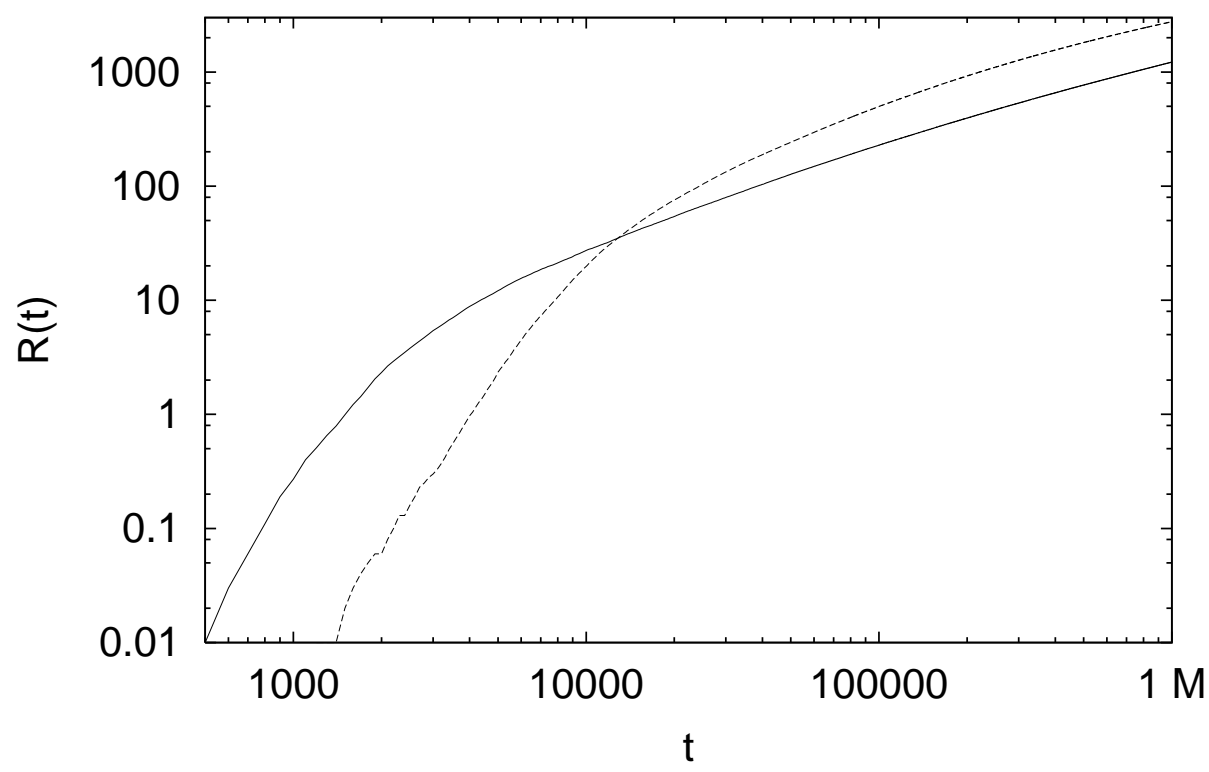

Figure 6: Total number of visits to the origin, up to the time $t$, in Model D. The results are averages over 100 realizations for $P=0.1$ and $m=5$ (solid line) and 10 (dashed line). 

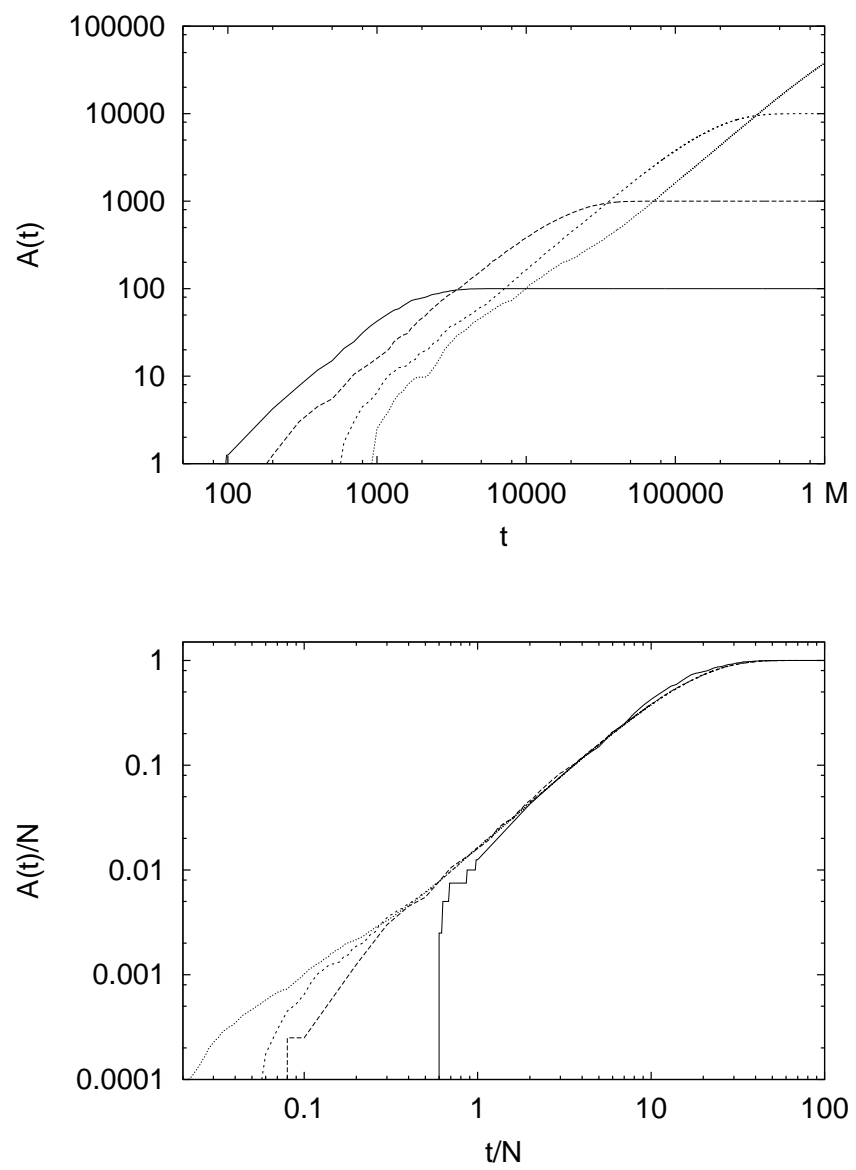

Figure 7: Top: Number of sites that are accessible to the random walkers at time $t$ in Model D for, from bottom to top, network sizes $N=10^{2}, 10^{3}, 10^{4}$, and $10^{5}$ (one realization with four walkers). Bottom: Scaled results of those shown at the top. Both figures are for $P=0.1$ and $m=5$. 


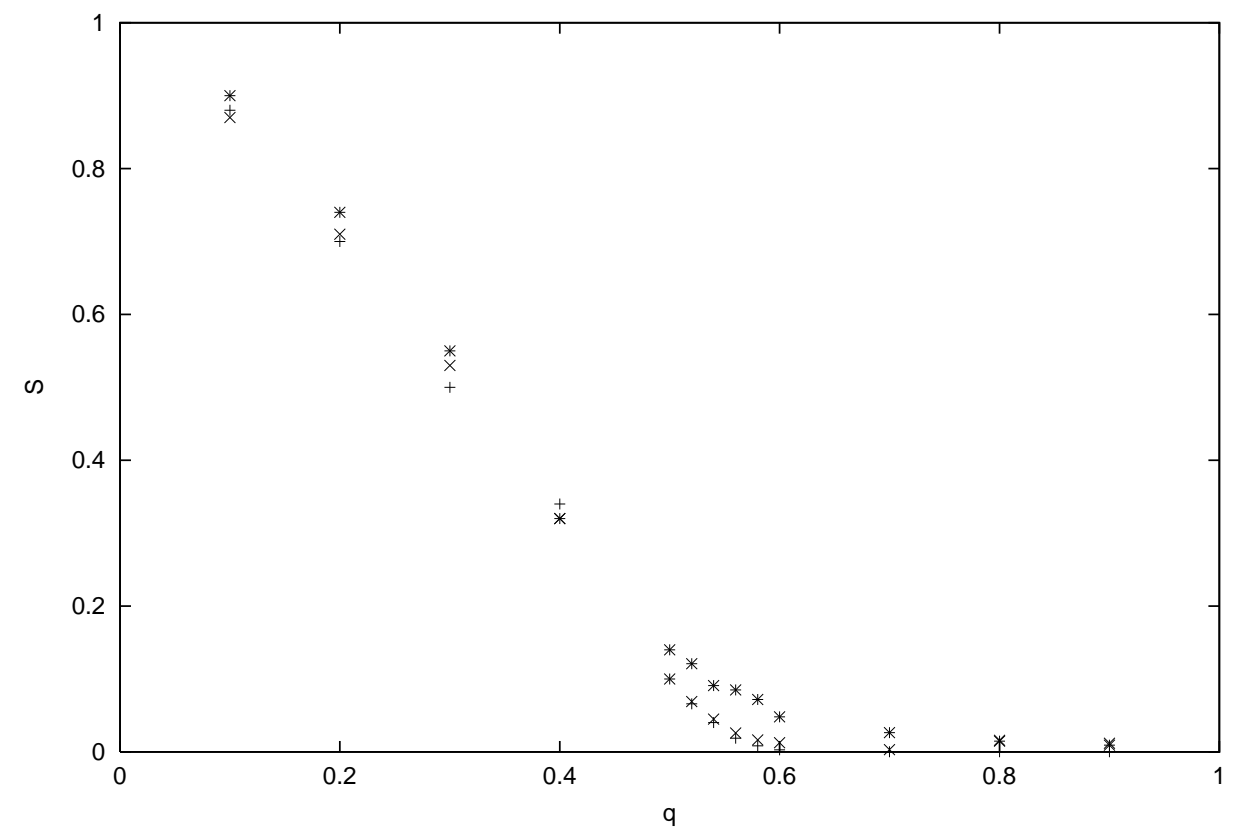

Figure 8: Final fraction of visited sites, when a fraction $q$ of the $n$ network sites is removed; the removed $q N$ sites are the first who joined the network; $m=1, t \leq 10^{7}, P=0.1$. The network size is $N=10^{4}(+), 10^{3}(\times), 10^{2}$ (stars). The results for $m=5$ do not differ much. 